\title{
A Taxonomy of Technologies for Fingerprint-Based Indoor Localization
}

\author{
Mário Andrade Vieira de Melo Neto ${ }^{1}$, Gibeon Soares de Aquino Júnior ${ }^{1}$ \\ ${ }^{1}$ Departamento de Matemática e Informática Aplicada \\ Universidade Federal do Rio Grande do Norte (UFRN) \\ Natal - RN - Brazil \\ mariovmelo@gmail.com, gibeon@dimap.ufrn.br
}

\begin{abstract}
In recent years, the need for indoor localization has increased. Earlier systems have been deployed in order to demonstrate that indoor localization can be done. Many researchers are referring to location estimation as a crucial component in numerous applications. The indoor localization techniques can be classified using the following classes: proximity, fingerprint, triangulation and vision analysis, with the fingerprint class being the most used. This paper presents the results of a literature systematic mapping on fingerprint-based indoor localization aiming to identify the technologies used for this purpose. The selected search strategy returned 1003 papers, which underwent a series of inclusion and exclusion criteria that resulted with 539 articles being accepted. This work identified that the main technology used for indoor localization is the WIFI, followed by ZigBee. As novelty, we propose a taxonomy of technologies used in the context of fingerprint-based indoor localization.
\end{abstract}

\section{Introduction}

Indoor localization systems have become very popular in recent years. These systems provide a new automation layer for the localization of people or objects in indoor environments, which makes them crucial for many applications. According to [Lymberopoulos et al. 2015] after more than one decade in this area, the indoor localization problem remains unsolved. There does not seem to exist a technology or a combination of technologies can solve this problem in an acceptable manner and at a low cost.

For outdoor location, the most popular technology is the Global Positioning System (GPS) [Kaplan and Hegarty 2005], which works based on satellites, making it quite accurate in external locations but inappropriate for indoor spaces. This limitation is caused by the inability of the satellite's signals to propagate in areas that are full of obstacles, causing failures or the impossibility to calculate the target's position. Aiming to achieve the same success as the GPS, indoor localization systems has been increasingly gaining space, providing new strategies for the detection of people and objects. There are many real world situations in which these systems can be used, such as: detection and control of products stored in a warehouse, location of medical personnel or equipments in a hospital, location of firemen in a building on fire, location of police dogs trained to find explosives in a building and finding tagged maintenance tools and equipment scattered all over a plant [Liu et al. 2007]. 
Currently, large companies [Google 2014, Apple 2015] are investing in research and development of solutions for indoor localization. Nevertheless, there is still no localization solution proven effective on indoor environments at the same scale that GPS is for outdoors. One of the reasons for this is the high complexity of indoor environments, which are always associated with a number of challenges such as the influence of obstacles (walls, equipment and people), overlap of signals emitted by various types of equipments present in the locations, variety of buildings types and dimensions that are considered small when compared to outdoors.

According to [Gu et al. 2009], the indoor localization techniques are classified using the following classes: triangulation, proximity, fingerprint and vision analysis. The fingerprint technique was chosen for this study because according to [Farid et al. 2013, Bolliger 2008], is the most widely used approach for indoor localization. [Kaemarungsi and Krishnamurthy 2004], the fingerprint-based indoor localization is defined as the determination of a position through the process of mapping the environment's aspects, such as the strength of the received signal, the magnetic field present at a location or any other characteristic that can identify a position. With the result of this mapping and the position where it was done, it is possible to make an inference to get approximate location of people or objects without the need of any specialized equipment.

This paper aims to perform a literature review on the fingerprint-based indoor localization subject to create a taxonomy of technologies. Therefore, a systematic mapping was performed using the guidelines defined by [Petersen et al. 2008, Kitchenham and Brereton 2013]. The purpose of this review was to identify the most used technologies, the types of researches that are being conducted and the resultant contributions to the area.

The rest of the paper is organized as follows: Section 2 describes the protocol used to perform the mapping, therefore it presents research questions, search terms used, classification scheme and paper selection process. Section 3 presents the main results and their implications. On Section 4 we discuss and analyze the found results. Section 5 concludes the study and indicates future trends on the subject.

\section{Research Methodology}

In this paper, we present a systematic mapping review based on guide written by [Petersen et al. 2008]. Figure 1 shows an overview of the systematic mapping process used in this study.

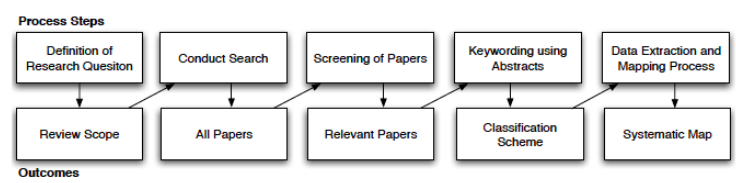

Figure 1. Systematic Mapping Process defined by [Petersen et al. 2008]

Following the process, the first step was to define the research questions, which are presented as follow: Which technologies are used in fingerprint-based indoor localization? (RQ1); How are the papers distributed over time?(RQ2); In the papers found, which types of researches were used? (RQ3); What are the main type of contribution described in each work? ( $R Q 4)$. 


\subsection{Search Strategy}

The research started by identifying the key terms used in the proposed subject. For this, several searches were conducted on the research databases in order to identify possible synonyms and keywords that could return the highest number of relevant papers. As a result of these pilot searches, the following terms were chosen:

\section{("indoor location" OR "indoor localization" OR "indoor positioning”) AND (fin- gerprint)}

Our search strategy used the most well-known academic work databases in the science computer area. In order to obtain all the relevant works, we used the meta-search engine Scopus ${ }^{1}$, since it covers all the sources that are relevant to our study. It was performed a solo search resulting with 1003 papers for evaluation.

\subsection{Inclusion and Exclusion Criteria}

Every recovered paper was manually evaluated using a set of criteria in order to identify whether it would be included or not in the mapping. For this purpose, we evaluated title, abstract, keywords and, when necessary, introduction and conclusion.

The inclusion criteria used to indicate whether a paper would be part of the mapping or not are: propose or evaluate an indoor localization technology and the paper was already reported, only the latest will be considered.

For a paper to be excluded from the mapping, it needed to fit into at least one criterion as follows: papers not written in English, papers in which the contribution is not involved with the area of computer science, Papers that do not have full versions available.

\subsection{Establishing a Classification Scheme}

Only a few classifications on fingerprint-based indoor localization have been done in the past. However, some of them were not based on an overview of the literature, but on industrial cases as [Deak et al. 2012] presents; or covered only one part of the subject, as described in [Liu et al. 2007] and [Farid et al. 2013]. Therefore, there is a risk that some important categories have not been addressed. Thus, in order to perform an accurate and complete classification, we used the keywording process defined by [Mujtaba et al. 2008].

The keywording process is performed in two stages. In the first stage, the reviewers read abstracts in search of keywords and concepts that reflect the main contribution of the article and the technologies used in the research. While doing so, the reviewer also identifies the research context. When this is done, a set of keywords from different papers are clustered and combined to develop a high level classification. In papers where the abstracts were too poor in quality to allow the extraction of meaningful keywords, reviewers chose to study the introduction or the conclusion sections of the paper.

\subsection{Selection Process}

This stage of the protocol was divided into two phases. First, we applied the inclusion and exclusion criteria, which resulted in papers that were relevant for the mapping process. Table 1 shows this result. The second phase was responsible for analyzing and classifying the papers based on the definition of the categories identified during the development of the classification system described in Section 2.5.

\footnotetext{
${ }^{1}$ http://www.scopus.com
} 
Table 1. Selection papers Stages

\begin{tabular}{|r|l|r|}
\hline Stage & Description & n \\
\hline 1 & Identified relevant papers & 1003 \\
\hline 2 & Excluded inaccessible papers & 1003 \\
\hline 3 & Excluded based on language & 996 \\
\hline 4 & Excluded duplicated papers & 954 \\
\hline 5 & Excluded based on title & 946 \\
\hline 6 & Excluded based on abstract & 829 \\
\hline 7 & Relevant papers & 539 \\
\hline
\end{tabular}

In the process of selection and classification of works, no inclusion criteria using quality levels were applied. This way, we tried to avoid the discard of studies relevant to the research because we could compromise the overview of the area, which we wish to obtain.

\subsection{Classification Scheme}

The papers were classified based on three different facets. Each facet consists of a set of categories in which papers can be mapped. The facets are: technology, main contribution and research type.

Technology Facet: Determines the technologies used in the research. This classification was obtained through the key wording process. Figure 4 presents this result.

Contribution Facet:This classification determines the main type of contribution achieved by the researcher. In other words, the improvements proposed for the subject. These contributions have been obtained using the keywording process and are presented in Table 2.

Table 2. Contribution Type Facet

\begin{tabular}{|l|l|}
\hline Category & Description \\
\hline Solution & $\begin{array}{l}\text { Represents a software or computational solution. Also apply to this } \\
\text { definition: tool, system or application. }\end{array}$ \\
\hline Method & $\begin{array}{l}\text { Indicates how things should be done, i.e., using Bluetooth to perform } \\
\text { the indoor localization. Algorithms, techniques and approaches are } \\
\text { part of this classification. }\end{array}$ \\
\hline Scheme & $\begin{array}{l}\text { Describes a plan or protocol to treat specific problems. Defines a set } \\
\text { of procedures and rules for the research or proposed solution. }\end{array}$ \\
\hline Metric & Metrics and measures for indoor localization. \\
\hline Model & $\begin{array}{l}\text { Represents a mathematical model description for indoor localiza- } \\
\text { tion. }\end{array}$ \\
\hline
\end{tabular}

Research Type Facet: This classification was suggested by [Wieringa et al. 2006] and defines six categories which are briefly described in Table 3.

\subsection{Data Extraction}

During this phase, all necessary data for our mapping study of the 1003 papers obtained in stage 1 of the selection process was extracted based on a predefined extraction form. 
Table 3. Research Type Facet

\begin{tabular}{|l|l|}
\hline Category & Description \\
\hline Validation Research & $\begin{array}{l}\text { Techniques investigated are novel and have not yet been im- } \\
\text { plemented in practice. Techniques used are for example ex- } \\
\text { periments. }\end{array}$ \\
\hline Evaluation Research & $\begin{array}{l}\text { Techniques are implemented in practice and an evaluation of } \\
\text { the technique is conducted. This also includes to identify } \\
\text { problems in industry. }\end{array}$ \\
\hline Solution Proposal & $\begin{array}{l}\text { A solution for a problem is proposed, the solution can be } \\
\text { either novel or significant extension of an existing technique. } \\
\text { The potential benefits and the applicability of the solution is } \\
\text { shown by an example or a good line of argumentation. }\end{array}$ \\
\hline Philosophical Papers & $\begin{array}{l}\text { These papers sketch a new way of looking at existing things } \\
\text { by structuring the field in form of a taxonomy or conceptual } \\
\text { framework. }\end{array}$ \\
\hline Opinion Papers & $\begin{array}{l}\text { These papers express the personal opinion of somebody } \\
\text { whether a certain technique is good or bad, or how things } \\
\text { should been done. }\end{array}$ \\
\hline Experience Papers & $\begin{array}{l}\text { What and how something has been done in practice. It has } \\
\text { to be the personal experience of the author. }\end{array}$ \\
\hline
\end{tabular}

This form allowed the extraction of all data with all of the details needed for the research questions analysis. Since our focus was to obtain a taxonomy of technologies used for indoor localization, the data extraction was performed individually for each paper.

\section{Results of Literature Mapping}

In order to answer RQ2, Figure 2 presents the number of included papers separated by year, with the higher value occurring in 2013 with 141 papers. We noticed a small decrease in the amount of included papers in 2014. This fact can be explained because the mapping execution took place in November/2014, so many papers were still not available in the research databases. It is noticed that in the last three years, the featured subject has received more attention, having had, in the 2012-2014 period, an increase of $40 \%$ in the number of papers when compared to the 2004-2011 period.

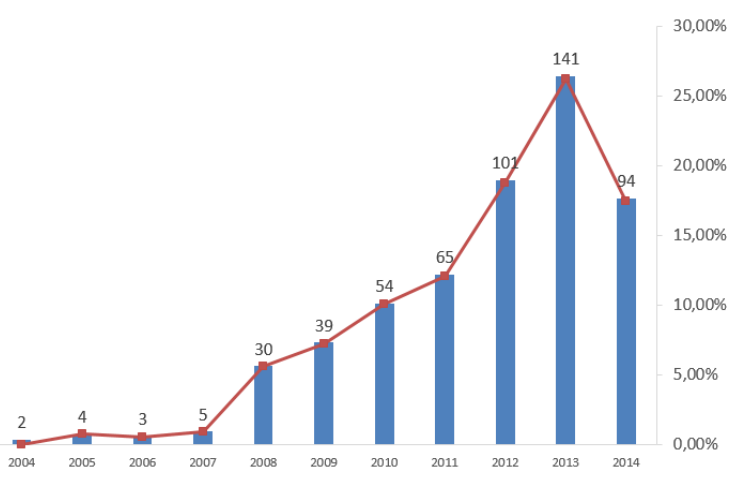

Figure 2. Included Papers per Year 
Figure 3(a) presents the distribution of the classified papers in the research type facet defined in Section 2.5. The obtained results answer RQ3 and demonstrate that most papers - about $90 \%$ of the total report solution proposals. The numbers of validation and evaluation researches represent together approximately $10 \%$ of the total of researches done. This demonstrates the low amount of researches for the validation of solution proposals in laboratories or in the industry.

In order to answer RQ4, we present, in Figure 3(b), the amount of papers for each main contribution defined. This classification was obtained using the key wording process described in Section 2.3. The numbers for solutions and methods contributions represent more than $97 \%$ of the total, which demonstrates that the researcher's focus are on the pursuit of "how" to make the indoor localization of objects or persons. Of this total, the methods represent more than $63 \%$ of all papers. This expressive number can be explained due to sub-categories grouping such as algorithms, techniques and approaches in a higher-level category.

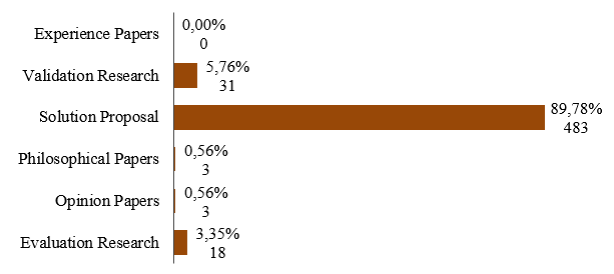

(a) Distribution of research types

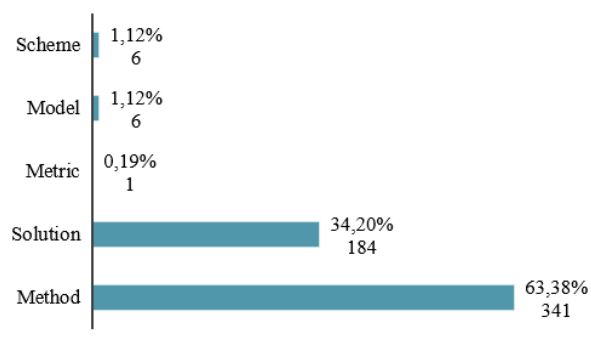

(b) Distribution of contribution types

Figure 3. Research and contribution types distribution

Figure 4 presents the required data for answer the RQ1, including the technologies used in the evaluated papers and their quantities. We also organize the data obtained into a taxonomy of technologies based on the transmission medium employed for spreading the information. It is noticed that the number of technologies used exceeds the number of papers evaluated because, in some cases, more than one technology has been used in the research. Among all, the technology that was mostly used was the WIFI, which surpassed more than 6 times the second place. According to [Research 2009], in 2012 about 1.5 billion devices were activated using with WIFI. In addition to this, another fact should be taken into consideration: the cost. Since the infrastructure needed exists practically everywhere, it would not be necessary to modify or insert any equipment, therefore reducing costs.

Another technology that deserves to be mentioned because of the number of researches in which it was used is the ZigBee. Despite being very similar to WIFI and Bluetooth, it proposes better power management and low data transmission [Hung et al. 2010]. Despite these features, there are some factors that prevent ZigBee to be used in large scale, such as high cost to deploy and short range. According to [Research 2013] the Bluetooth technology will be present in almost 4 billion devices being 1 billion of this total on smartphones in 2016. So it was expected a much larger number for this technology. Since we expected that, it would be at least among the top five. This technology has some advantages for indoor positioning as presents [Svalastog 2007], however [Farid et al. 2013] presents one characteristic may have direct influence in the presented numbers of using 


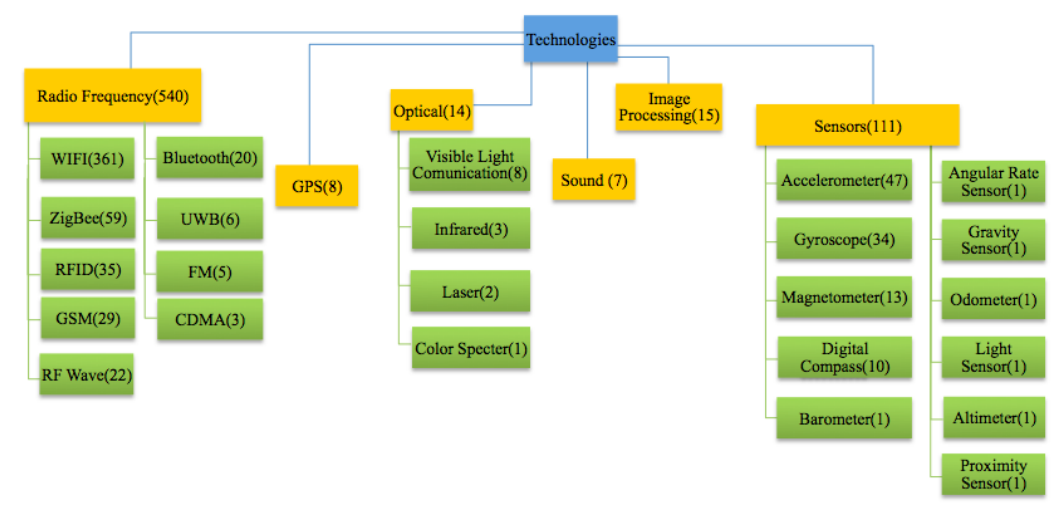

Figure 4. Taxonomy of Technologies

Bluetooth in localization is that, in each location finding, it runs the device discovery procedure; due to this, it significantly increases the localization latency $(10-30 \mathrm{~s})$ and power consumption as well. For this reason, the Bluetooth technology has a major issue to overcome when it comes to realtime positioning applications.

The Sensors category's has gained a lot of attention in recent years in the area and the technologies responsible for it are undoubtedly accelerometers and gyroscopes. This large increase is directly linked to the Smartphone popularization process. According to [Gartner 2012], 1.75 billion people have Smartphones with advanced capabilities. These Smartphones with advanced capabilities typically have multiple sensors, such as accelerometers and gyroscopes, which are the most used in researches in the Sensors category, indicating that there is still a large gap for this theme when compared to the number of papers in the Radio Frequency category.

\section{Discussion}

Performing this mapping study, we realize that the indoor localization problem is still a hot topic and is not solved. It was evident, and according to [Lymberopoulos et al. 2015] there is no solution that can recreate the experience that GPS offers outdoors in the indoor environment. The results presented demonstrate that Solution Proposal research type overcome sum of all the others research types. This fact demostrate the intense effort of researchers in obtaining a standardized solution for the indoor localization problem.In relation to the technologies, the WI FI is most used in research and has much higher numbers related the others and one of the reasons for this is researchers attempt to obtain same standardization obtained with the GPS. However, this standardization has not been achieved due to indoor environments having many factors that could affect localization obtainment such as furniture, walls and even people.

By analyzing the list of technologies obtained, we realized that several studies focus on more than one technology at a time. Figure 5 presents the rate of hybrid approaches found in the evaluated papers compared to the number of included papers per year. For a better analysis, a ratio line linking the two measures is presented. We noticed that between 2004 and 2007, no research was performed using combined technologies. Since 2008, researchers began to discreetly use hybrid approaches, which are responsible for about $9 \%$ of all of the papers written in the period; the use of hybrid approaches remained stable until 2012, having a 1\% decrease in 2009. From 2012, we noticed a gradual 
growth with a constant rate of $2 \%$ a year. Despite the low number of researches with this characteristic, there is a tendency that, in the upcoming years, this number will grow and new solutions and methods using combined technologies will be proposed. We believe that one of the reasons that led the researchers to use this type of approach is the fact that indoor environments can be very complex and that no single technology is able to satisfactorily adapt itself to these environments complexities in order to perform an accurate localization.

Other taxonomies have been proposed but not focused on presenting an overview of the use of technologies for locating systems, particularly those fingerprint-based. In [Hightower and Borriello 2001] taxonomy is presented focused on analyzing location solutions. Several evaluation properties have Been listed: precision, accuracy, scale, cost, and limitations. In the work of [Kjærgaard 2007] defines a taxonomy for location fingerprinting systems from standard properties such as scale, output, measurements, and roles. Moreover, in an article describing the survey of wireless indoor positioning, [Liu et al. 2007] have developed a taxonomy for performance of wireless indoor positioning focused on only one property and disregarding other types of technologies.

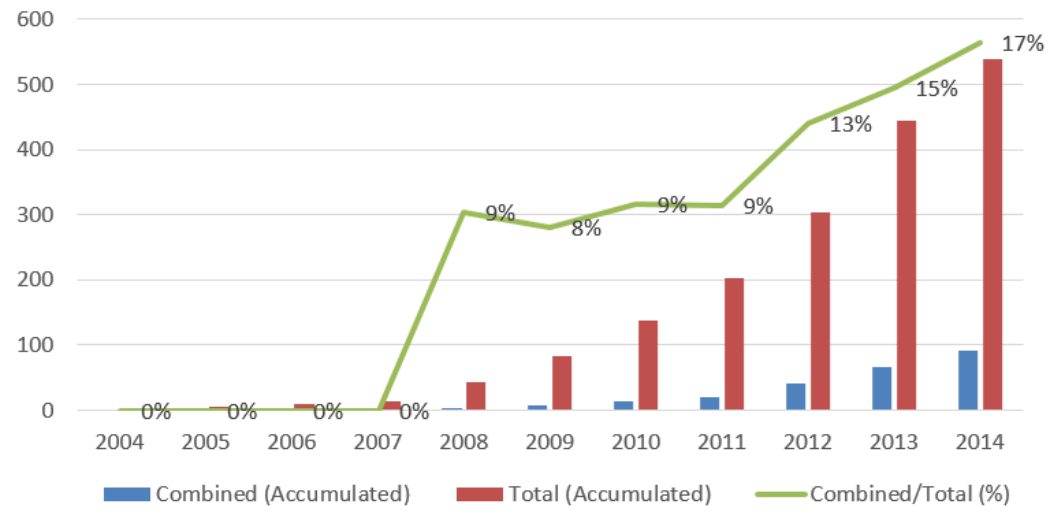

Figure 5. Combined tecnology use evolution

\section{Conclusion}

In this paper, we report the results of a systematic mapping study on the subject of fingerprint-based indoor localization. The collection and interpretation of data related to this context produced a number of important discoveries, which allow us to understand the evolution of this area in recent years and also point trends and open issues. In addition to the results obtained from the data analysis, we created taxonomy for the technologies used in the context of fingerprint-based indoor localization.

Initially, this mapping showed that the most used type of research is the proposed solution, which demonstrates the pursuit for an indoor localization solution. Another finding is the confirmation of the WIFI technology as the most used in researches performed on the focus area, which confirms our expectations since in fact it is the most disseminated and present technology in most locations.

Another finding presented by this mapping was the increase on the number of papers that use a set of technologies in their research. The most promising category on this matter is the Sensors, which represents $86 \%$ of the reviewed papers. On the other 
hand, the Radio Frequency category obtained only $15 \%$, which is mostly due to the WIFI technology, which is normally used in an isolated way.

As novelty, we propose on this paper a taxonomy of technologies used in the indoor localization fingerprint-based area. This finding has identified and grouped technologies based on the transmission medium employed for spreading the information. Although this mapping was performed for one indoor localization class, we believe that this taxonomy can be extended to other indoor localization classes.

Based on the achieved results, we notice the increasing use of Sensors in the proposed solutions, which might lead to a key role in future solutions. The new generations of Smartphones have been showing the market an integration with new and different Sensors. Since the localization in indoor environments is more complex than in the outdoors, there is a tendency for the new solutions to agglutinate different technologies and approaches. For this reason, we believe that hybrid solutions are the future of indoor localization, creating new opportunities for scientific and technological researches.

As a contribution, this study is intend to assist researchers providing an overview of the indoor location area and the technologies used by others researchers. This insight may help on current and new researches on the area. As future work, we pretend to perform a systematic mapping on the others indoor localization classes.

\section{Acknowledgment}

This work was partially supported by the National Institute of Science and Technology for Software Engineering (INES) funded by CNPq under grant 573964/2008-4

\section{References}

Apple (2015). Footprint: Indoor positioning with core location, http://developer.apple.com/library/ios/samplecode/footprint/.

Bolliger, P. (2008). Redpin-adaptive, zero-configuration indoor localization through user collaboration. In Proceedings of the first ACM international workshop on Mobile entity localization and tracking in GPS-less environments, pages 55-60. ACM.

Deak, G., Curran, K., and Condell, J. (2012). A survey of active and passive indoor localisation systems. Computer Communications, 35(16):1939-1954.

Farid, Z., Nordin, R., and Ismail, M. (2013). Recent advances in wireless indoor localization techniques and system. Journal of Computer Networks and Communications, 2013.

Gartner (2012). Gartner says worldwide mobile phone sales declined 1.7 percent in 2012, http://www.gartner.com/newsroom/id/2335616.

Google (2014). Indoor maps, http://www.google.com/intl/ptbr/maps/about/partners/indoormaps/.

Gu, Y., Lo, A., and Niemegeers, I. (2009). A survey of indoor positioning systems for wireless personal networks. Communications Surveys \& Tutorials, IEEE, 11(1):13-32.

Hightower, J. and Borriello, G. (2001). Location systems for ubiquitous computing. Computer, (8):57-66. 
Hung, M.-H., Lin, S.-S., Cheng, J.-Y., and Chien, W.-L. (2010). A zigbee indoor positioning scheme using signal-index-pair data preprocess method to enhance precision. pages 548-553.

Kaemarungsi, K. and Krishnamurthy, P. (2004). Properties of indoor received signal strength for wlan location fingerprinting. Proceedings of MOBIQUITOUS 2004 - 1st Annual International Conference on Mobile and Ubiquitous Systems: Networking and Services, pages 14-23.

Kaplan, E. and Hegarty, C. (2005). Understanding GPS: principles and applications. Artech house.

Kitchenham, B. and Brereton, P. (2013). A systematic review of systematic review process research in software engineering. Information and Software Technology, 55(12):2049_ 2075.

Kjærgaard, M. B. (2007). A taxonomy for radio location fingerprinting. In Location-and Context-Awareness, pages 139-156. Springer.

Liu, H., Darabi, H., Banerjee, P., and Liu, J. (2007). Survey of wireless indoor positioning techniques and systems. IEEE Transactions on Systems, Man and Cybernetics Part C: Applications and Reviews, 37(6):1067-1080.

Lymberopoulos, D., Giustiniano, D., Lenders, V., Rea, M., Marcaletti, A., et al. (2015). A realistic evaluation and comparison of indoor location technologies: Experiences and lessons learned. ACM/IEEE International Conference on Information Processing in Sensor Networks, pages 178-189.

Mujtaba, S., Petersen, K., Feldt, R., and Mattsson, M. (2008). Software product line variability: A systematic mapping study. School of Engineering, Blekinge Inst. of Technology.

Petersen, K., Feldt, R., Mujtaba, S., and Mattsson, M. (2008). Systematic mapping studies in software engineering. In 12th International Conference on Evaluation and Assessment in Software Engineering, volume 17, page 1.

Research, A. (2009). Wi-fi ic market share analysis and forecasts, https://www.abiresearch.com/market-research/service/wi-fi.

Research, A. (2013). Emerging bluetooth verticals, http://www.bluetooth.org.

Svalastog, M. S. (2007). Indoor Positioning-Technologies, Services and Architectures. $\mathrm{PhD}$ thesis, UNIVERSITY OF OSLO.

Wieringa, R., Maiden, N., Mead, N., and Rolland, C. (2006). Requirements engineering paper classification and evaluation criteria: a proposal and a discussion. Requirements Engineering, 11(1):102-107. 